NISSUNA UMANA INVESTIGAZIONE SI PUO DIMANDARE VERA SCIENZIA S'ESSA NON PASSA PER LE MATEMATICHE DIMOSTRAZIONI LEONARDO DA VINCI

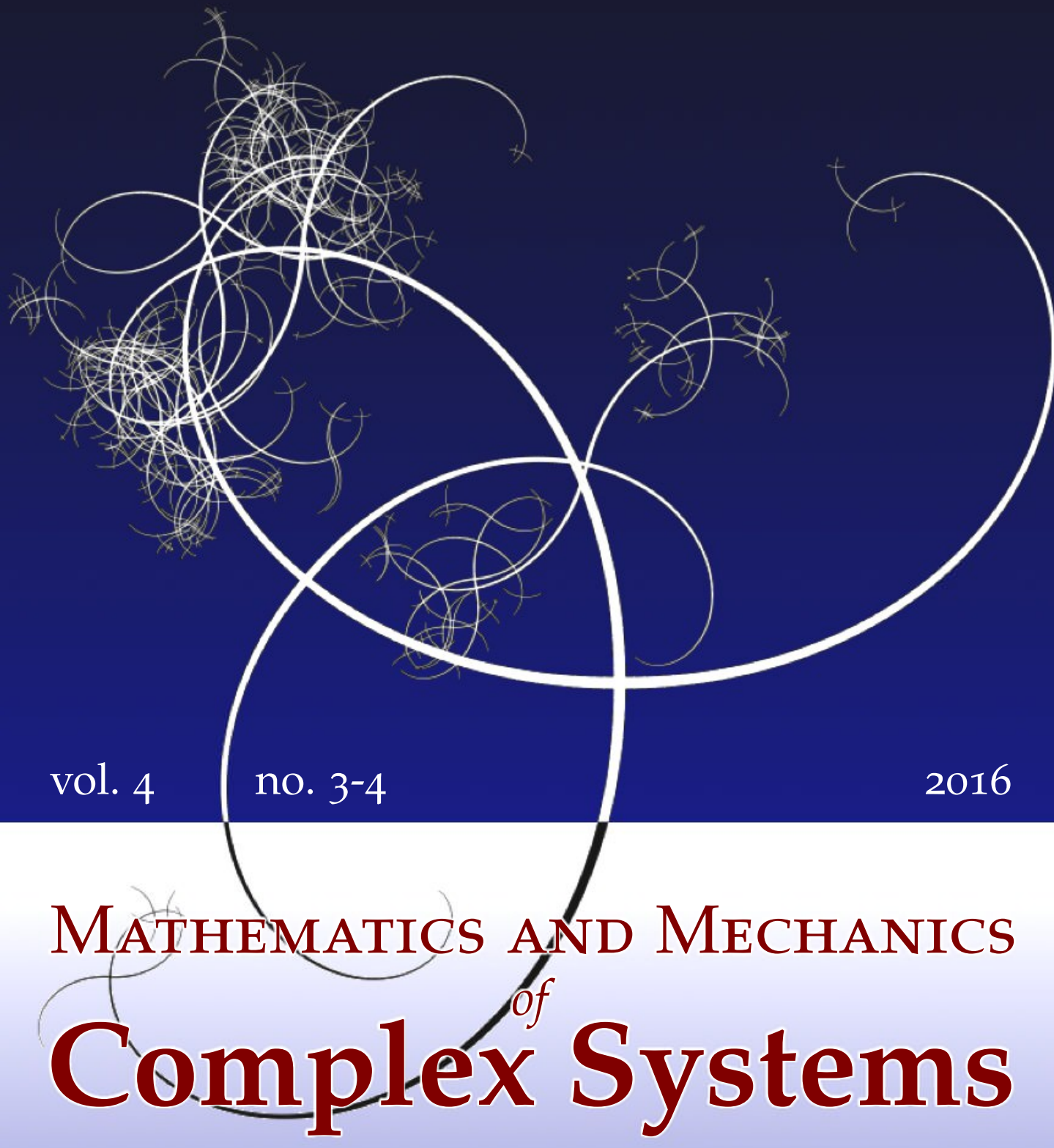

Massimo Campanino and Michele Gianfelice SOME RESULTS ON THE ASYMPTOTIC BEHAVIOR OF FINITE CONNECTION PROBABILITIES IN PERCOLATION 


\title{
SOME RESULTS ON THE ASYMPTOTIC BEHAVIOR OF FINITE CONNECTION PROBABILITIES IN PERCOLATION
}

\author{
Massimo Campanino And Michele Gianfelice
}

\begin{abstract}
We review results of two previous papers on the asymptotic behavior of finite connection probabilities in three or more dimensions for Bernoulli percolation and the Fortuin-Kasteleyn random-cluster model. In the introduction, we prove a multidimensional renewal theorem that is needed for these results and previous results on Ornstein-Zernike behavior; the proof is significantly simpler than that originally derived by Doney (1966) and those of other subsequent works on this subject.
\end{abstract}

\section{Introduction}

In the last few decades, much progress has been made in the rigorous study of the asymptotic behavior of connection functions in percolation outside the critical point. This problem is related to that of typical fluctuations of clusters and, in two dimensions, of interfaces [Gallavotti 1972; Greenberg and Ioffe 2005].

In the case of the subcritical regime for Bernoulli percolation or the FortuinKasteleyn (FK) random-cluster model on a regular lattice, connection functions, i.e., the probabilities that two points are connected, decay exponentially as the distance between the points tends to infinity [Grimmett 1999; 2006]. In [Campanino and Ioffe 2002; Campanino et al. 2008], the exact asymptotic behavior of this decay has been established. It is called Ornstein-Zernike behavior [1914] since they derived it in statistical mechanics for systems outside the critical point. Moreover, in [Campanino and Ioffe 2002; Campanino et al. 2008], the strict convexity with positive Gaussian curvature and the analyticity of equidecay surfaces were established.

An extension of the techniques developed in [Campanino and Ioffe 2002] has been developed in [Campanino and Gianfelice 2009], where the exact asymptotics

\section{Communicated by Raffaele Esposito.}

Campanino and Gianfelice are partially supported by the Gruppo Nazionale per l'Analisi Matematica, la Probabilità e le loro Applicazioni (GNAMPA) and thank the referee for useful suggestions.

MSC2010: 60K35, 82B43, 60K15, 60F17.

Keywords: random-cluster model, Ornstein-Zernike behavior for connectivities, renormalization,

Ruelle operator, local limit theorem, invariance principle. 
for the probability that three points are connected has been derived. We refer the reader to [Coquille et al. 2014] for an application of this result.

In this paper, we review some recent results related to the case of finite connections in the supercritical regime. Here an infinite cluster exists and ordinary connection functions between two points converge to a positive constant as their distance tends to infinity. Indeed, in order to be connected between each other, it is enough for them to be both connected to the infinite cluster that is spread everywhere. One is therefore led to consider finite connection functions, i.e., the probabilities that two points are connected between each other but not to the infinite cluster. In this case, the points are surrounded by a surface in the dual lattice, where plaquettes of the dual lattice are occupied when the dual bond is vacant. One can expect in three or more dimensions that these surfaces have similar fluctuations as the connecting clusters in the subcritical regime and therefore that finite connection functions also exhibit Ornstein-Zernike behavior. The situation in two dimensions, where the dual surface is basically composed of two random paths that can freely fluctuate with the only constraint being no intersection, is different. In this case, for Bernoulli percolation on the square lattice above the critical point, [Campanino et al. 2010] established that finite connection functions exhibit a different behavior, corresponding to that of two independent random paths.

Here we expose some recent results on the asymptotic behavior of finite connection functions in three or more dimensions that appeared in [Campanino and Gianfelice 2011; 2015]. These results refer both to Bernoulli percolation and to the FK random-cluster model in the highly supercritical regime, i.e., when the parameter $p$ related to the occupation probability of a bond is close to 1 . It would be desirable to extend the validity of the results to a larger region of parameter values.

1.1. Notation. We denote $|x|:=\sum_{i=1}^{d}\left|x_{i}\right|$, by $\langle\cdot, \cdot\rangle$ the scalar product in $\mathbb{R}^{d}$ and by $\|\cdot\|:=\sqrt{\langle\cdot, \cdot\rangle}$ the associated Euclidean norm. We then set, for $x \neq 0$, $\hat{x}:=x /\|x\|$ and $\mathbb{S}^{d-1}:=\left\{z \in \mathbb{R}^{d}:\|z\|=1\right\}$, and denoting by $B$ the closed unit ball in $\mathbb{R}^{d}$, for $r>0$, we let $r B:=\left\{x \in \mathbb{R}^{d}:\|x\| \leq r\right\}$ and $B_{r}(x):=x+r B$.

For any $t \in \mathbb{R}^{d}$, we define

$$
\mathscr{H}_{x}^{t}:=\left\{y \in \mathbb{R}^{d}:\langle t, y\rangle=0\right\}
$$

to be the $(d-1)$-dimensional hyperplane in $\mathbb{R}^{d}$ orthogonal to the vector $t$ passing through $x$.

In the sequel, we will omit the dependence on $x$ in the notation if the point is taken to be the origin.

1.2. A note on the multidimensional renewal process. We first consider a simple model of a "multidimensional renewal process" that, with suitable adaptations, is at the basis of the arguments used in most of these works. 
Let $\left\{X_{i}\right\}_{i \geq 1}$ be a sequence of independent and identically distributed random vectors with values in $\mathbb{Z}^{d}$. We assume that $\mathbb{E}\left[e^{t\left|X_{1 \mid}\right|}\right]<\infty$ for some $t>0$. Moreover, we assume that the support of the law of $X_{1}$ spans all of $\mathbb{Z}^{d}$ and that $\mu:=\mathbb{E}\left[X_{1}\right]$ is such that $\mu \neq 0$.

The exact asymptotics of the expected number of visits to a point $x \in \mathbb{Z}^{d}$ by the random walk just defined, as $\|x\|$ tends to infinity, was derived for the first time in [Doney 1966] under the hypothesis of the existence of the first $l$ moments, with $l$ an explicit function of the lattice dimensions. Extensions of this result to the renewal measure of $x+B$, with $B$ a bounded subset of $\mathbb{R}^{d}$, are given in [Stam 1969], as well as in [Carlsson and Wainger 1984] where the exponential decay of the tail of $X_{1}$ is also assumed.

Here, under this last assumption, we present a proof of the multidimensional theorem on which Theorem 1 relies; the proof is considerably simpler than that originally derived in [Doney 1966].

Under the hypotheses just given on the law of $X_{1}$, the following properties are satisfied:

Local limit theorem: There exists $\alpha \in\left(0, \frac{1}{2}\right)$ such that, uniformly in $x \in \mathbb{Z}^{d}$ with $\|x-n \mu\|<n^{1 / 2+\alpha}$ as $n$ tends to infinity,

$$
\mathbb{P}\left\{\sum_{i=1}^{n} X_{i}=x\right\}=\frac{\exp \left\{-\left\langle A^{-1}(x-n \mu),(x-n \mu)\right\rangle / 2 n\right\}}{\sqrt{(2 \pi n)^{d} \operatorname{det} A}}(1+o(1)),
$$

where $A$ is the covariance matrix of $X_{1}$.

Large deviation estimate from the mean: This follows from the exponential tail of the distribution of $X_{1}$.

To estimate the asymptotic behavior in $n$ of the series

$$
\sum_{k \geq 1} \mathbb{P}\left\{\sum_{i=1}^{k} X_{i}=n \mu\right\}
$$

we decompose it as

$$
\begin{aligned}
& \sum_{k \geq 1} \mathbb{P}\left\{\sum_{i=1}^{k} X_{i}=n \mu\right\}= \sum_{k=1}^{n-\left\lfloor n^{1 / 2+\alpha}\right\rfloor} \mathbb{P}\left\{\sum_{i=1}^{k} X_{i}=n \mu\right\} \\
&+\sum_{k=n-\left\lfloor n^{1 / 2+\alpha}\right\rfloor+1}^{n+\left\lfloor n^{1 / 2+\alpha}\right\rfloor-1} \mathbb{P}\left\{\sum_{i=1}^{k} X_{i}=n \mu\right\} \\
&+\sum_{k \geq n+\left\lfloor n^{1 / 2+\alpha}\right\rfloor} \mathbb{P}\left\{\sum_{i=1}^{k} X_{i}=n \mu\right\} .
\end{aligned}
$$


Then we estimate the second term on the right-hand side by using the local limit theorem estimate and the sum of the first and the third terms by using the large deviation upper bound.

Therefore, we get

$$
\begin{aligned}
& \sum_{k \in \mathbb{N}:|k-n|<n^{1 / 2+\alpha}} \frac{\exp \left\{-\left\langle A^{-1}(k \mu-n \mu),(k \mu-n \mu)\right\rangle / 2 k\right\}}{\sqrt{(2 \pi k)^{d} \operatorname{det} A}}(1+o(1)) \\
& =\sum_{k \in \mathbb{N}:|k-n|<n^{1 / 2+\alpha}} \frac{\exp \left\{-|k-n|^{2}\left\langle A^{-1} \mu, \mu\right\rangle /\left(2 n\left(1+O\left(n^{\alpha-1 / 2}\right)\right)\right)\right\}}{\sqrt{\left(2 \pi n\left(1+O\left(n^{\alpha-1 / 2}\right)\right)^{d} \operatorname{det} A\right.}}(1+o(1)) \\
& =\frac{1}{\sqrt{(2 \pi n)^{d-1}(\operatorname{det} A)\left\langle A^{-1} \mu, \mu\right\rangle}}(1+o(1)) .
\end{aligned}
$$

Moreover, by the standard large deviation estimate for the sums of independent and identically distributed random variables with exponential tails, we obtain

$$
\sum_{k \in \mathbb{N}:|k-n| \geq n^{1 / 2+\alpha}} \mathbb{P}\left\{\sum_{i=1}^{k} X_{i}=n \mu\right\} \leq e^{-c_{1} n^{2 \alpha}} .
$$

Summing up (7) and (8),

$$
\sum_{k \geq 1} \mathbb{P}\left\{\sum_{i=1}^{k} X_{i}=n \mu\right\}=\frac{c_{2}}{n^{(d-1) / 2}}(1+o(1)),
$$

with $c_{2}$ a positive constant.

1.3. Possible developments. It would be desirable to extend the results of [Campanino and Gianfelice 2011;2015] to values of $p$ larger than some critical $p_{c}$. Moreover, it should be possible to extend the results of [Campanino et al. 2010] from independent percolation to the FK random-cluster model.

\section{Ornstein-Zernike behavior for the finite connectivity function in highly supercritical percolation models}

2.1. Bernoulli percolation and the FK random-cluster model. Let $\mathbb{L}^{d}$ denote the graph associated to $\left(\mathbb{Z}^{d}, \mathbb{E}^{d}\right)$, with

$$
\mathbb{E}^{d}:=\left\{\{x, y\} \in \mathscr{P}_{2}\left(\mathbb{Z}^{d}\right):|x-y|=1\right\} .
$$

Let $\mathfrak{L}_{0}$ be the collection of subgraphs of $\llbracket^{d}$ of finite order. If $G \in \mathfrak{L}_{0}, V(G)$ is the set of vertices and $E(G)$ is the set of edges of $G$. We denote by $\bar{G}$ the graph 
induced by the union of $V(G)^{1}$ with the sets of vertices of the components of $\llbracket^{d} \backslash G$ of finite size. We define the boundary of $G$ as the set

$$
\partial G:=\left\{e \in \mathbb{E}^{d} \backslash E(G):|e \cap V(G)|=1\right\} \subset \mathbb{E}^{d}
$$

and the external boundary of $G$ to be $\bar{\partial} G:=\partial \bar{G}$.

Considering the realization of $\mathbb{L}^{d}$ as a geometric graph embedded in $\mathbb{R}^{d}$, which with abuse of notation we still denote by $\mathbb{L}^{d}$, we can look at it as a cell complex, i.e., as the union of $\mathbb{Z}^{d}$ and $\mathbb{E}^{d}$ representing the collections of 0 -cells and of 1-cells, respectively. We denote by $\left(\mathbb{Z}^{d}\right)^{*}$ the collection of $d$-cells dual to 0 -cells in $\mathbb{L}^{d}$, that is, the collection of Voronoi cells of $\mathbb{L}^{d}$, and by $\left(\mathbb{E}^{d}\right)^{*}$ the collection of $(d-1)$-cells dual to 1-cells in $\mathbb{\llbracket}^{d}$, usually called plaquettes in the physics literature.

A bond percolation configuration on $\mathbb{L}^{d}$ is a map $\mathbb{E}^{d} \ni e \mapsto \omega_{e} \in\{0,1\}$. Setting $\Omega:=\{0,1\}^{\mathbb{E}^{d}}$, we define

$$
\Omega \ni \omega \mapsto \boldsymbol{E}(\omega):=\left\{e \in \mathbb{E}^{d}: \omega_{e}=1\right\} \in \mathscr{P}\left(\mathbb{E}^{d}\right) .
$$

Denoting by $\mathbb{G}:=\left\{G \subseteq \mathbb{L}^{d}: G=G(E), E \in \mathscr{P}\left(\mathbb{E}^{d}\right)\right\}$ the collection of spanning subgraphs of $\mathbb{L}^{d}$, we define the random graph

$$
\Omega \ni \omega \mapsto \boldsymbol{G}(\omega):=G(\boldsymbol{E}(\omega)) \in \mathbb{G}
$$

and by $\kappa(\omega)$ the number of its components. Then, given $l \geq 1$ and $x_{1}, \ldots, x_{l} \in \mathbb{Z}^{d}$, we denote by

$$
\Omega \ni \omega \mapsto \boldsymbol{C}_{\left\{x_{1}, \ldots, x_{l}\right\}}(\omega) \in \mathscr{P}\left(\mathbb{Z}^{d}\right)
$$

the common open cluster of the points $x_{1}, \ldots, x_{l} \in \mathbb{Z}^{d}$, that is, the set of vertices of the component of the random graph $\boldsymbol{G}$ to which these points belong, provided it exists, and set

$$
\Omega \ni \omega \mapsto \boldsymbol{E}_{\left\{x_{1}, \ldots, x_{l}\right\}}(\omega):=E\left(\boldsymbol{C}_{\left\{x_{1}, \ldots, x_{l}\right\}}(\omega)\right) \cap \boldsymbol{E}(\omega) \subseteq \mathbb{E}^{d} .
$$

We also define, in case $\boldsymbol{C}_{\left\{x_{1}, \ldots, x_{l}\right\}}$ is finite, the random set $\bar{\partial} \boldsymbol{C}_{\left\{x_{1}, \ldots, x_{l}\right\}}$ to be equal to $\bar{\partial} G$ if $G$ is the component of $\boldsymbol{G}$ whose set of vertices is $\boldsymbol{C}_{\left\{x_{1}, \ldots, x_{l}\right\}}$ and the random set

$$
\boldsymbol{S}_{\left\{x_{1}, \ldots, x_{l}\right\}}:=\left(\bar{\partial} \boldsymbol{C}_{\left\{x_{1}, \ldots, x_{l}\right\}}\right)^{*} .
$$

Let $\mathscr{F}$ be the $\sigma$-algebra generated by the cylinder events of $\Omega$. If $\Lambda$ is a finite subset of $\mathbb{Z}^{d}$, let $\mathbb{E}^{\Lambda}$ be the subset of $\mathbb{E}^{d}$ such that $V\left(\mathbb{E}^{\Lambda}\right)=\Lambda$ and denote $\Omega_{\Lambda}:=$ $\{0,1\}^{\mathbb{E}^{\Lambda}}$, by $\mathscr{F}_{\Lambda}$ the corresponding product $\sigma$-algebra and by $\mathscr{T}_{\Lambda}$ the $\sigma$-algebra generated by the cylinder events $\left\{\omega \in \Omega: \omega_{\Delta} \in A\right\}$, where $\Delta \subset \Lambda^{c}$ and $A \in \mathscr{F}_{\Delta}$. The random-cluster measures on $\mathbb{Z}^{d}$ [Fortuin and Kasteleyn 1972; Edwards and Sokal

${ }^{1}$ We refer the reader to [Campanino and Gianfelice 2011; 2015] for the notions of graph theory needed in the study of percolation theory. 
1988] with parameters $q \geq 1$ and $p \in[0,1]$ are the dependent bond percolation probability measures $\mathbb{P}$ on $(\Omega, \mathscr{F})$ specified by

$$
\mathbb{P}\left(A \mid \mathscr{T}_{\Lambda}\right)=\mathbb{P}_{\Lambda ; q, p}(A) \mathbb{P}-\text { a.s., } \quad A \in \mathscr{F},
$$

where, setting, for any $\pi \in \Omega_{\Lambda}^{c}, \Omega_{\Lambda}^{\pi}:=\left\{\omega \in \Omega: \omega_{e}=\pi_{e}, e \in \mathbb{E}^{d} \backslash \mathbb{E}^{\Lambda}\right\}, \mathbb{P}_{\Lambda ; q, p}^{\pi}$ is the probability measure on $(\Omega, \mathscr{F})$ with density

$$
\mathbb{P}_{\Lambda ; q, p}^{\pi}(\omega):=\frac{1}{\mathscr{E}_{\Lambda}^{\pi}(q ; p)} p^{|\boldsymbol{E}(\omega)|}(1-p)^{\left|\mathbb{E}^{\Lambda} \backslash \boldsymbol{E}(\omega)\right|} q^{\kappa_{\Lambda}(\omega)} \mathbf{1}_{\Omega_{\Lambda}^{\pi}}(\omega),
$$

where $\kappa_{\Lambda}(\omega)$ is the number of the components of $\boldsymbol{G}(\omega)$ intersecting $\Lambda$.

For $q=1$, the FK random-cluster model coincides with Bernoulli percolation. Therefore, we omit in this case the value of $q$ in the specification of $\mathbb{P}$.

Random-cluster measures satisfy the FKG inequality; that is, for any couple $f, g$ of random variables increasing with respect to the natural partial order defined on $\Omega, \mathbb{P}(f g) \geq \mathbb{P}(f) \mathbb{P}(g)$. Moreover, the partial order of $\Omega$ induces a stochastic ordering on the elements of the collection of probability measures defined by (18); namely, for any increasing random variable $f, \mathbb{P}_{\Lambda ; q, p}^{\pi_{1}}(f) \leq \mathbb{P}_{\Lambda ; q, p}^{\pi_{2}}(f)$ if $\pi_{1} \leq \pi_{2}$. Hence, denoting by $\preceq$ such an ordering, for all $\pi \in \Omega_{\Lambda}^{c}$, $\mathbb{P}_{\Lambda ; q, p}^{\mathrm{f}} \preceq \mathbb{P}_{\Lambda ; q, p}^{\pi} \preceq \mathbb{P}_{\Lambda ; q, p}^{\mathrm{W}}$, where $\mathbb{P}_{\Lambda ; q, p}^{\mathrm{f}}$ and $\mathbb{P}_{\Lambda ; q, p}^{\mathrm{W}}$ stand for the probability measure with density (18) corresponding to the free $(\pi \equiv 0)$ and to the wired $(\pi \equiv 1)$ boundary conditions, respectively. Since, for \# =f, w, the (weak) limit of the sequence $\left\{\mathbb{P}_{\Lambda ; q, p}^{\#}\right\}$ along any exhaustion $\{\Lambda\} \uparrow \mathbb{Z}^{d}$ exists (see, e.g., [Grimmett 2006, Theorem 4.19]) and is the random-cluster measure that we denote by $\mathbb{P}_{q, p}^{\#}$, the ordering $\preceq$ extends as well to random-cluster measures and $\mathbb{P}_{q, p}^{\mathrm{f}} \preceq \mathbb{P} \preceq \mathbb{P}_{q, p}^{\mathrm{W}}$.

Furthermore, denoting by $\mathbb{P}_{p^{\prime}}:=\mathbb{P}_{1, p^{\prime}}$ the independent Bernoulli bond percolation probability measures on $\mathbb{Z}^{d}$ with parameter $p^{\prime}$, by Theorem 3.21 of [Grimmett 2006, p. 43], we obtain the stochastic domination inequalities

$$
\mathbb{P}_{p(q)} \preceq \mathbb{P}_{q, p}^{\mathrm{f}} \preceq \mathbb{P}_{q, p}^{\mathrm{w}} \preceq \mathbb{P}_{p},
$$

where $p(q):=p /(p+q(1-p))$.

In the following, we assume the random-cluster measure $\mathbb{P}_{q, p}$ to be translationinvariant.

\subsection{Results.}

Theorem 1. For any $d \geq 3$ and any $q \geq 1$, there exists $p_{0}=p_{0}(q, d)$ such that, for all $p>p_{0}$, uniformly in $x \in \mathbb{Z}^{d}$ as $\|x\| \rightarrow \infty$,

$$
\mathbb{P}_{q, p}\left\{0 \leftrightarrow x,\left|\boldsymbol{C}_{\{0, x\}}\right|<\infty\right\}=\frac{\Phi_{q, p}(\hat{x})}{\sqrt{(2 \pi\|x\|)^{d-1}}} e^{-\tau_{q, p}(x)}(1+o(1)),
$$


where $\Phi_{q, p}$ is a positive real analytic function on $\mathbb{S}^{d-1}$ and $\tau_{q, p}$ an equivalent norm in $\mathbb{R}^{d}$.

As a byproduct of the proof, we also obtain the following result.

Theorem 2. For any $d \geq 3$ and any $q \geq 1$, there exists $p_{0}=p_{0}(q, d)$ such that, for all $p>p_{0}$, the equidecay set of the two-point finite connectivity function is locally analytic and strictly convex. Moreover, the Gaussian curvature of the equidecay set is uniformly positive.

These theorems are proven for the independent percolation case in [Campanino and Gianfelice 2011] and for the general case in [Campanino and Gianfelice 2015].

2.3. Analysis of connectivities. The following result appears as Proposition 4 in [Campanino and Gianfelice 2015].

Proposition 3. Given $q \geq 1$ and $p \in(0,1)$, let $\mathbb{P}_{q, p}$ be a translation-invariant random-cluster measure on $\mathbb{Z}^{d}$ with parameters $q$ and $p$. Then for any $x \in \mathbb{R}^{d}$,

$$
\tau_{q, p}(x):=-\lim _{n \rightarrow \infty} \frac{1}{n} \log \mathbb{P}_{q, p}\left\{0 \leftrightarrow\lfloor n x\rfloor,\left|\boldsymbol{C}_{\{0,\lfloor n x\rfloor\}}\right|<\infty\right\}
$$

exists and is a convex and homogeneous-of-order-1 function on $\mathbb{R}^{d}$.

Proof. For any $\Delta \subseteq \mathbb{Z}^{d}$, let us denote by $\boldsymbol{E}_{\Delta}:=\bigcup_{x \in \Delta} \boldsymbol{E}_{\{x\}} \subseteq \mathbb{E}^{d}$ the set of edges belonging to open paths starting at the vertices of $\Delta$.

Now let $\Lambda$ be a finite subset of $\mathbb{Z}^{d}$ such that $\Lambda \ni 0$. For any two distinct lattice points $x, y \in \Lambda$, looking at $\mathbf{1}_{\left\{0 \leftrightarrow x, 0 \leftrightarrow \Lambda^{c}\right\}}$ and $\mathbf{1}_{\left\{x \leftrightarrow y, y \leftrightarrow \Lambda^{c}\right\}}$ as functions of $\left(\boldsymbol{E}_{\{x\}}, \boldsymbol{E}_{\Lambda^{c}}\right)$, they are both nondecreasing on $\boldsymbol{E}_{\{x\}}$ and nonincreasing on $\boldsymbol{E}_{\Lambda^{c}}$. Therefore, by Theorem 2.1 in [van den Berg et al. 2006],

$$
\begin{aligned}
& \mathbb{P}_{q, p}\left(\left\{0 \leftrightarrow x, 0 \leftrightarrow \Lambda^{c}\right\} \cap\left\{x \leftrightarrow y, y \leftrightarrow \Lambda^{c}\right\} \mid\left\{x \leftrightarrow \Lambda^{c}\right\}\right) \\
& \geq \mathbb{P}_{q, p}\left(\left\{0 \leftrightarrow x, 0 \leftrightarrow \Lambda^{c}\right\} \mid\left\{x \leftrightarrow \Lambda^{c}\right\}\right) \mathbb{P}_{q, p}\left(\left\{x \leftrightarrow y, y \leftrightarrow \Lambda^{c}\right\} \mid\left\{x \leftrightarrow \Lambda^{c}\right\}\right) ;
\end{aligned}
$$

that is,

$$
\begin{aligned}
\mathbb{P}_{q, p}\left\{x \leftrightarrow \Lambda^{c}\right\} \mathbb{P}_{q, p}\left\{0 \leftrightarrow x, x \leftrightarrow y, x \leftrightarrow \Lambda^{c}\right\} \\
\\
\quad \geq \mathbb{P}_{q, p}\left\{0 \leftrightarrow x, x \leftrightarrow \Lambda^{c}\right\} \times \mathbb{P}_{q, p}\left\{x \leftrightarrow y, x \leftrightarrow \Lambda^{c}\right\},
\end{aligned}
$$

which implies

$$
\begin{aligned}
\mathbb{P}_{q, p}\left\{0 \leftrightarrow x, x \leftrightarrow y, \boldsymbol{C}_{\{0, x, y\}} \cap \Lambda^{c}=\varnothing\right\} \\
\\
\quad \geq \mathbb{P}_{q, p}\left\{0 \leftrightarrow x, \boldsymbol{C}_{\{0, x\}} \cap \Lambda^{c}=\varnothing\right\} \times \mathbb{P}_{q, p}\left\{x \leftrightarrow y, \boldsymbol{C}_{\{x, y\}} \cap \Lambda^{c}=\varnothing\right\} .
\end{aligned}
$$

But

$$
\mathbb{P}_{q, p}\left\{0 \leftrightarrow y, \boldsymbol{C}_{\{0, y\}} \cap \Lambda^{c}=\varnothing\right\} \geq \mathbb{P}_{q, p}\left\{0 \leftrightarrow x, x \leftrightarrow y, \boldsymbol{C}_{\{0, x, y\}} \cap \Lambda^{c}=\varnothing\right\}
$$


hence,

$$
\begin{aligned}
\mathbb{P}_{q, p}\{0 & \left.\leftrightarrow y, \boldsymbol{C}_{\{0, y\}} \cap \Lambda^{c}=\varnothing\right\} \\
& \geq \mathbb{P}_{q, p}\left\{0 \leftrightarrow x, \boldsymbol{C}_{\{0, x\}} \cap \Lambda^{c}=\varnothing\right\} \times \mathbb{P}_{q, p}\left\{x \leftrightarrow y, \boldsymbol{C}_{\{x, y\}} \cap \Lambda^{c}=\varnothing\right\} .
\end{aligned}
$$

Taking the limit $\Lambda \uparrow \mathbb{Z}^{d}$,

$$
\begin{aligned}
\mathbb{P}_{q, p}\left(\left\{0 \leftrightarrow y,\left|\boldsymbol{C}_{\{0, y\}}\right|<\infty\right\}\right) \\
\quad \geq \mathbb{P}_{q, p}\left(\left\{0 \leftrightarrow x,\left|\boldsymbol{C}_{\{0, x\}}\right|<\infty\right\}\right) \times \mathbb{P}_{q, p}\left(\left\{x \leftrightarrow y,\left|\boldsymbol{C}_{\{x, y\}}\right|<\infty\right\}\right) .
\end{aligned}
$$

Proceeding as in the proof of Proposition 15 in [Campanino and Gianfelice 2011], we obtain the thesis.

2.4. The independent percolation case. We will show, without going too much into details for which we refer the reader to [Campanino and Gianfelice 2011], how the proof of Theorem 1 in the independent percolation case can be reduced to that of an estimate of the form presented in (9).

Given $x, y \in \mathbb{Z}^{d}$, we set

$$
\varphi(x, y):=\left\{\begin{array}{cc}
\min \left\{\left|S_{\{x, y\}}(\omega)\right|: \omega \in\{x \leftrightarrow y\}\right\}, & x \neq y, \\
0, & x=y .
\end{array}\right.
$$

Here $\varphi$ is symmetric and translation-invariant, so we write $\varphi(x, y)=\varphi(x-y)$.

We remark that, given $G_{i}:=\left(V_{i}, E_{i}\right), i=1,2$, two connected subgraphs of $\mathbb{L}^{d}$ of finite size, by (11), $\partial\left(G_{1} \cup G_{2}\right) \subseteq \partial G_{1} \cup \partial G_{2}$. Moreover,

$$
\bar{\partial}\left(G_{1} \cup G_{2}\right)=\partial\left(\overline{G_{1} \cup G_{2}}\right) \subseteq \partial \overline{G_{1}} \cup \partial \overline{G_{2}} .
$$

This allowed us to prove (see Lemma 4 and Proposition 5 in [Campanino and Gianfelice 2011]) that the sequence $\left\{\bar{\varphi}_{n}\right\}_{n \in \mathbb{N}}$, where, for any $n \in \mathbb{N}, \mathbb{R}^{d} \ni x \mapsto \bar{\varphi}_{n}(x):=$ $\varphi(\lfloor n x\rfloor) / n \in \mathbb{R}^{+}$, converges pointwise to $\bar{\varphi}$, which is a convex, homogeneous-oforder-1 function on $\mathbb{R}^{d}$. Furthermore, $\left\{\bar{\varphi}_{n}\right\}_{n \in \mathbb{N}}$ converges uniformly on $\mathbb{S}^{d-1}$.

Conjecture 4. We conjecture that $\bar{\varphi}$ is the $l_{1}$ norm in $\mathbb{R}^{d}$, i.e., $\bar{\varphi}(x)=|x|:=$ $\sum_{i=1}^{d}\left|x_{i}\right|$.

In the limit of $p$ tending to 1 , the support of the probability distribution of the finite cluster containing two sites $x$ and $y$, conditioned to its existence, is given by the configurations minimizing $\varphi(x-y)$. Therefore, given $p$ sufficiently close to 1 , we can estimate the probability that the finite cluster of two points has an external boundary of size larger than $(1+\delta) \varphi(x-y)$, for $\delta$ larger than a given value $\delta^{*}(p, d)$ that tends to 0 as $p$ tends to 1 . Indeed, we have:

Proposition 5. There exist $c_{3}=c_{3}(d)>1$ and $\delta^{*}=\delta^{*}(p, d)$, with $\lim _{p \uparrow 1} \delta^{*}(p, d)=$ 0 , such that, for any $p \in\left(1-1 / c_{3}, 1\right)$ and any $\delta>\delta^{*}$,

$$
\mathbb{P}_{p}\left(\left\{\left|\boldsymbol{S}_{\{0, x\}}\right| \geq(1+\delta) \varphi(x)\right\} \mid\left\{\left|\boldsymbol{C}_{\{0, x\}}\right|<\infty\right\}\right) \leq c_{4} e^{-c_{5} \varphi(x)},
$$


with $c_{4}=c_{4}(d, p)>1$ and $c_{5}=c_{5}(d, p, \delta)>0$.

Hence, we are left with the estimate of the probability that the external boundary of $\boldsymbol{C}_{\{0, x\}}$ has size smaller than $(1+\delta) \varphi(x)$ for $\delta>\delta^{*}$.

Renewal structure of connectivities. Given $t \in \mathbb{S}^{d-1}$, we define

$$
\mathscr{H}_{y}^{t}:=\left\{x \in \mathbb{R}^{d}:\langle t, x\rangle=\langle t, y\rangle\right\}, \quad y \in \mathbb{R}^{d},
$$

to be the $(d-1)$-dimensional hyperplane in $\mathbb{R}^{d}$ orthogonal to the vector $t$ passing through a point $y \in \mathbb{R}^{d}$ and the corresponding half-spaces

$$
\begin{aligned}
& \mathscr{H}_{y}^{t,-}:=\left\{x \in \mathbb{R}^{d}:\langle t, x\rangle \leq\langle t, y\rangle\right\}, \\
& \mathscr{H}_{y}^{t,+}:=\left\{x \in \mathbb{R}^{d}:\langle t, x\rangle \geq\langle t, y\rangle\right\} .
\end{aligned}
$$

Let $t \in \mathbb{S}^{d}$. Given two points $x, y \in \mathbb{Z}^{d}$ such that $\langle x, t\rangle \leq\langle y, t\rangle$, we denote by $\boldsymbol{C}_{\{x, y\}}^{t}$ the cluster of $x$ and $y$ inside the strip $\mathscr{S}_{\{x, y\}}^{t}:=\mathscr{H}_{x}^{t,+} \cap \mathscr{H}_{y}^{t,-}$ provided it exists.

Let $u$ be the first of the unit vectors in the direction of the coordinate axes $u_{1}, \ldots, u_{d}$ such that $\langle t, u\rangle$ is maximal

Definition 6. Given $t \in \mathbb{S}^{d-1}$, let $x, y \in \mathbb{Z}^{d}$ such that $\langle x, t\rangle \leq\langle y, t\rangle$ be connected in $\mathscr{S}_{\{x, y\}}^{t}$. The points $b \in \boldsymbol{C}_{\{x, y\}}^{t}$ such that

(1) $\langle t, x+u\rangle \leq\langle t, b\rangle \leq\langle t, y-u\rangle$ and

(2) $\boldsymbol{C}_{\{x, y\}}^{t} \cap \mathscr{S}_{\{b-u, b+u\}}^{t}=\{b-u, b, b+u\}$

are said to be $t$-break points of $\boldsymbol{C}_{\{x, y\}}$. The collection of such points, which we remark is a totally ordered set with respect to the scalar product with $t$, will be denoted by $\boldsymbol{B}^{t}(x, y)$.

Definition 7. Given $t \in \mathbb{S}^{d-1}$, let $x, y \in \mathbb{Z}^{d}$ such that $\langle x, t\rangle \leq\langle y, t\rangle$ be connected in $\mathscr{S}_{\{x, y\}}^{t}$. An edge $\{b, b+u\}$ such that $b, b+u \in \boldsymbol{B}^{t}(x, y)$ is called a $t$-bond of $\boldsymbol{C}_{\{x, y\}}$. The collection of such edges will be denoted by $\boldsymbol{E}^{t}(x, y)$ while $\boldsymbol{B}_{e}^{t}(x, y) \subset$ $\boldsymbol{B}^{t}(x, y)$ will denote the subcollection of $t$-break points $b$ of $\boldsymbol{C}_{\{x, y\}}$ such that the edge $\{b, b+u\} \in \boldsymbol{E}^{t}(x, y)$.

Definition 8. Given $t \in \mathbb{S}^{d-1}$, let $x, y \in \mathbb{Z}^{d}$ such that $\langle t, x\rangle \leq\langle t, y\rangle$ be connected. Then $x, y \in \mathbb{Z}^{d}$ are said to be $h_{t}$-connected if

(1) $x$ and $y$ are connected in $\mathscr{Y}_{\{x, y\}}^{t}$ and $\left|\boldsymbol{C}_{\{x, y\}}^{t}\right|<\infty$ and

(2) $x+u, y-u \in \boldsymbol{B}^{t}(x, y)$.

Moreover, denoting by $\left\{x \stackrel{h_{t}}{\leftrightarrow} y\right\}$ the event that $x$ and $y$ are $h_{t}$-connected, we set

$$
h_{t}^{(p)}(x, y):=\mathbb{P}_{p}\left\{x \stackrel{h_{t}}{\leftrightarrow} y\right\} .
$$


Notice that, by translation invariance, $h_{t}^{(p)}(x, y)=h_{t}^{(p)}(y-x, 0)$, so in the sequel, we will denote it simply by $h_{t}^{(p)}(y-x)$. We also define by convention $h_{t}^{(p)}(0)=1$.

Definition 9. Let $t \in \mathbb{S}^{d-1}$ and $x, y \in \mathbb{Z}^{d}$ be $h_{t}$-connected. If $\boldsymbol{B}^{t}(x+u, y-u)$ is empty, then $x$ and $y$ are said to be $f_{t}$-connected and the corresponding event is denoted by $\left\{x \stackrel{f_{t}}{\leftrightarrow} y\right\}$. We then set

$$
f_{t}^{(p)}(y-x):=\mathbb{P}_{p}\left\{x \stackrel{f_{t}}{\leftrightarrow} y\right\} .
$$

We define by convention $f_{t}^{(p)}(0)=0$.

Definition 10. Given $t \in \mathbb{S}^{d-1}$, let $x, y \in \mathbb{Z}^{d}$ such that $\langle t, x\rangle \leq\langle t, y\rangle$ be connected. Then:

(1) $x, y$ are called $\bar{h}_{t}$-connected and the corresponding event is denoted by $\left\{x \stackrel{\bar{h}_{t}}{\leftrightarrow} y\right\}$, if $\boldsymbol{C}_{\{x, y\}} \cap \mathscr{Y}_{\{y-u, y\}}^{t}=\{y-u, y\}$ and $\left|\boldsymbol{C}_{\{x, y\}} \cap \mathscr{H}_{y}^{t,-}\right|<\infty$.

(2) $x, y$ are called $\bar{f}_{t}$-connected and the corresponding event is denoted by $\left\{x \stackrel{\bar{f}_{t}}{\leftrightarrow} y\right\}$, if they are $\bar{h}_{t}$-connected and $\boldsymbol{B}^{t}(x, y)=\varnothing$.

Definition 11. Given $t \in \mathbb{S}^{d-1}$, let $x, y \in \mathbb{Z}^{d}$ such that $\langle t, x\rangle \leq\langle t, y\rangle$ be connected. Then:

(1) $x$ and $y$ are called $\tilde{h}_{t}$-connected, and the corresponding event is denoted by $\left\{x \stackrel{\tilde{h}_{t}}{\leftrightarrow} y\right\}$ if

(a) $\boldsymbol{C}_{\{x, y\}} \cap \mathscr{Y}_{\{x, x+u\}}^{t}=\{x, x+u\}$ and

(b) $\left|\boldsymbol{C}_{\{x, y\}} \cap \mathscr{H}_{x}^{t,+}\right|<\infty$.

(2) $x$ and $y$ are called $\tilde{f}_{t}$-connected, and the corresponding event is denoted by $\left\{x \stackrel{f_{t}}{\leftrightarrows} y\right\}$ if they are $\tilde{h}_{t}$-connected and $\boldsymbol{B}^{t}(x, y)=\varnothing$.

The functions $\bar{h}_{t}^{(p)}(x, y):=\mathbb{P}_{p}\left\{x \stackrel{\bar{h}_{t}}{\leftrightarrow} y\right\}$ and $\tilde{h}_{t}^{(p)}(x, y):=\mathbb{P}_{p}\left\{x \stackrel{\tilde{h}_{t}}{\leftrightarrow} y\right\}$ are translation invariant.

Denoting by $g_{t}^{(p)}(x, y)$, for $t \in \mathbb{S}^{d-1}$, the probability of the event

$$
\left\{x \stackrel{g_{t}}{\leftrightarrow} y\right\}:=\left\{x \leftrightarrow y,\left|\boldsymbol{C}_{\{x, y\}}\right|<\infty,\left|\boldsymbol{B}_{e}^{t}(x, y)\right| \leq 1\right\},
$$

which is also translation invariant, we obtain

$$
\begin{aligned}
\mathbb{P}_{p}\left\{0 \leftrightarrow x,\left|\boldsymbol{C}_{\{0, x\}}\right|<\infty\right\} & =g_{t}^{(p)}(x)+\sum_{z_{1}, z_{2} \in \mathbb{Z}^{d}} \bar{f}_{t}^{(p)}\left(z_{1}\right) h_{t}^{(p)}\left(z_{2}-z_{1}\right) \tilde{f}_{t}^{(p)}\left(x-z_{2}\right), \\
h_{t}^{(p)}(x) & =\sum_{z \in \mathbb{Z}^{d}} f_{t}^{(p)}(z) h_{t}^{(p)}(x-z) .
\end{aligned}
$$


Proposition 12. Given $t \in \mathbb{S}^{d-1}$, for any $p \in(0,1)$ and $x \in \mathbb{R}^{d}$ such that $\langle t, x\rangle>0$,

$$
\tau_{p}^{t}(x):=-\lim _{n \rightarrow \infty} \frac{1}{n} \log h_{t}^{(p)}(\lfloor n x\rfloor)
$$

exists and is a convex and homogeneous-of-order-1 function on $\mathbb{R}^{d}$. Moreover, for $p \in\left(1-1 / c_{3}, 1\right)$,

$$
\tau_{p}^{t}(x) \geq \bar{\varphi}(x) \log \frac{1}{c_{3}(1-p)} .
$$

The proof follows from the supermultiplicativity property of the function $h_{t}^{(p)}$; we refer the reader to Proposition 15 in [Campanino and Gianfelice 2011] for the details.

Since, by Proposition 3, for any $p \in(0,1)$ and $d \geq 2, \tau_{p}:=\tau_{1, p}$ is an equivalent norm in $\mathbb{R}^{d}$, there exists $c_{-}=c_{-}(p, d)>0$ such that

$$
\mathbb{P}_{p}\left\{0 \leftrightarrow x,\left|\boldsymbol{C}_{\{0, x\}}\right|<\infty\right\} \leq e^{-c_{-}\|x\|},
$$

while as a byproduct of the proof of Proposition 5 we get that there exists $c_{+}=$ $c_{+}(p, d)>0$ such that

$$
h_{t}^{(p)}(x) \geq e^{-c_{+}\|x\|},
$$

it follows that $\tau_{p}^{t} \geq \tau_{p}$ is finite and is an equivalent norm in $\mathbb{R}^{d}$.

Renormalization. We define

$$
\mathscr{W}:=\bigcap_{\hat{x} \in \mathbb{S}^{d-1}}\left\{w \in \mathbb{R}^{d}:\langle w, \hat{x}\rangle \leq \bar{\varphi}(\hat{x})\right\} .
$$

Given $x \in \mathbb{Z}^{d}$, let $t \in \mathfrak{d} W(x):=\{w \in \mathfrak{d} W:\langle w, x\rangle=\bar{\varphi}(x)\}$.

For $N \in \mathbb{N}$ larger than 1 , let us set $\mathfrak{t}_{N}=\mathfrak{t}_{N}(x):=\lfloor\|x\| / N\rfloor-1$ and

$$
\begin{aligned}
& y_{i}:=\lfloor i N \hat{x}\rfloor, \mathscr{H}_{i}^{t}:=\mathscr{H}_{y_{i}}^{t}, \mathscr{H}_{i}^{t,-}:=\mathscr{H}_{y_{i}}^{t,-}, \mathscr{H}_{i}^{t,+}:=\mathscr{H}_{y_{i}}^{t,+}, \quad i=0, \ldots, \mathfrak{t}_{N}, \\
& y_{\mathfrak{t}_{N}+1}:=x, \mathcal{H}_{y_{t_{N}+1}^{t}}^{t}:=\mathscr{H}_{x}^{t}, \mathcal{H}_{y_{\mathfrak{t}_{N}+1}^{t,-}}^{t}:=\mathcal{H}_{x}^{t,-}, \\
& \mathscr{S}_{i}^{t}:=\mathscr{H}_{i}^{t,+} \cap \mathscr{H}_{i+1}^{t,-} \text {. }
\end{aligned}
$$

With slight notational abuse, we still denote by $S_{\{0, x\}}$ its representation as a hypersurface in $\mathbb{R}^{d}$ and define

$$
\boldsymbol{C}_{i}^{t}:=\boldsymbol{C}_{\{0, x\}} \cap \mathscr{S}_{i}^{t}, \quad \boldsymbol{S}_{i}^{t}:=\boldsymbol{S}_{\{0, x\}} \cap \mathscr{S}_{i}^{t} .
$$

Hence, $\boldsymbol{C}_{\{0, x\}}=\bigcup_{i=0}^{\mathfrak{t}_{N}} \boldsymbol{C}_{i}^{t}$ and $\boldsymbol{S}_{\{0, x\}} \cap \mathcal{S}_{0, x}^{t} \subseteq \bigcup_{i=0}^{\mathrm{t}_{N}} \boldsymbol{S}_{i}^{t}$.

We call crossing any connected component $s$ of $\boldsymbol{S}_{i}^{t}$ such that, denoting by $\mathscr{K}(\boldsymbol{s})$ the compact subset of $\mathscr{S}_{i}^{t}$ whose boundary is $s$, there exist $y \in \mathscr{H}_{i}^{t,-} \cap \mathbb{Z}^{d}$ and $y^{\prime} \in \mathscr{H}_{i+1}^{t,+} \cap \mathbb{Z}^{d}$, both belonging to $\boldsymbol{C}_{\{0, x\}}$, which are connected by an open path in $\mathbb{L}^{d} \cap \mathscr{K}(\boldsymbol{s})$. 
We remark that, since $\boldsymbol{C}_{\{0, x\}}$ is connected, the existence of two crossings in $\mathcal{Y}_{i}^{t}$ implies the existence of two disjoint paths connecting $\mathscr{H}_{i}^{t}$ and $\mathscr{H}_{i+1}^{t}$ while the converse does not hold true in general.

We say that a slab $\mathscr{Y}_{i}^{t}$ is good if $\boldsymbol{S}_{i}^{t}$ is connected and made by just a single crossing of size smaller than twice the minimal one; otherwise, we call it bad.

In Section 3 of [Campanino and Gianfelice 2011], making use of a deterministic statement, we proved that the number of bad slabs is at most $2 \delta\|x\| / N$. Therefore, it is possible to modify the configuration of at most $c_{6} N^{d}$ bonds, with $c_{6}=$ $c_{6}(d, \delta)$, inside any 3 -tuple of consecutive slabs containing a single crossing in such a way that the resulting cluster will have at least one $t$-bond inside each of these slabs. Since these modifications can be performed independently, this fact and Proposition 5 imply the mass-gap condition $f_{t}^{(p)}(x) \leq e^{-c_{7}\|x\|} h_{t}^{(p)}(x)$ with $c_{7}=c_{7}(p)>0$, uniformly in $t \in \mathbb{S}_{x}^{d-1}$. Thus, from (37), we have $\tau_{p}^{t}=\tau_{p}$ for any $t \in \mathbb{S}_{x}^{d-1}$.

Extending $f_{t}^{(p)}$ to a function defined on the whole lattice by setting it equal to 0 , where it is undefined, set

$$
\begin{aligned}
& \mathbb{R}^{d} \ni s \mapsto H_{t}^{(p)}(s):=\sum_{x \in \mathbb{Z}^{d}} h_{t}^{(p)}(x) e^{\langle s, x\rangle} \in \overline{\mathbb{R}}, \\
& \mathbb{R}^{d} \ni s \mapsto F_{t}^{(p)}(s):=\sum_{x \in \mathbb{Z}^{d}} f_{t}^{(p)}(x) e^{\langle s, x\rangle} \in \overline{\mathbb{R}} .
\end{aligned}
$$

The renewal equation (38) implies

$$
H_{t}^{(p)}(s)=\frac{1}{1-F_{t}^{(p)}(s)} .
$$

Since (41) implies that, for all $p \in\left(p_{c}(d), 1\right)$, the effective domain of $H_{t}(s)$,

$$
\mathscr{D}_{t}^{p}:=\left\{s \in \mathbb{R}^{d}: H_{t}^{(p)}(s)<\infty\right\},
$$

is not empty since $\stackrel{\mathscr{D}}{t}_{t}^{p} \supseteq \mathscr{\Upsilon}_{t}^{p} \ni 0$, where

$$
\mathscr{K}_{t}^{p}:=\bigcap_{\hat{x} \in \mathbb{S}^{d-1}}\left\{s \in \mathbb{R}^{d}:\langle s, \hat{x}\rangle \leq \tau_{p}^{t}(\hat{x})\right\}
$$

is the convex body polar with respect to $u_{t}^{p}:=\left\{x \in \mathbb{R}^{d}: \tau_{t}^{p}(x) \leq 1\right\}$.

For $s \in \mathcal{K}_{t}^{p}$, since

$$
\langle s, x\rangle \leq \max _{s \in \mathscr{K}_{t}^{p}}\langle s, x\rangle=\tau_{p}^{t}(x) \leq 1,
$$

$h_{t}^{(p)}(x) \leq e^{-\tau_{p}^{t}(x)}$ and $F_{t}^{(p)}(s)$ is finite, moreover continuous, then, for all $s \in \partial \mathcal{K}_{t}^{p}$,

$$
\mathbb{Z}^{d} \ni x \mapsto q_{t ; s}^{(p)}(x):=f_{t}^{(p)}(x) e^{\langle s, x\rangle} \in \mathbb{R}
$$


is the density of the probability measure $Q_{t ; s}^{(p)}$ on $\left(\mathbb{Z}^{d}, \mathscr{B}\left(\mathbb{Z}^{d}\right)\right)$, which has exponentially decaying tails:

$$
f_{t}^{(p)}(x) e^{\langle s, x\rangle} \leq e^{-c_{7}\|x\|} h_{t}^{(p)}(x) e^{\langle s, x\rangle} \leq e^{-c_{7}\|x\|} .
$$

If $X$ is a random vector with probability distribution $Q_{t ; s}^{(p)}$, denoting by $\mathbb{E}_{p}^{t ; s}$ the expectation of a random variable under $Q_{t ; s}^{(p)}$, we set

$$
\mu_{t}^{p}(s):=\mathbb{E}_{p}^{t ; s}[X]=\operatorname{grad} \log F_{t}^{(p)}(s),
$$

while

$$
C_{t}^{p}(s):=\text { Hess } \log F_{t}^{(p)}(s)
$$

denotes the covariance matrix of $X$. Since $f_{t}^{(p)}(x)>0$ on a whole half-space, $C_{t}^{p}(s)$ is nondegenerate. Hence,

$$
\partial \mathcal{K}_{t}^{p}=\left\{s \in \mathbb{R}^{d}: F_{t}^{(p)}(s)=1\right\} \subseteq \mathbb{R}^{d} \backslash \mathscr{D}_{t}^{p}
$$

is a real analytic strictly convex surface with Gaussian curvature uniformly bounded away from 0 , and therefore, because $Q_{t ; s}^{(p)}$ is supported on $\mathscr{H}_{0}^{t,+} \cap \mathbb{Z}^{d}, \mu_{t}^{p}(s) \neq 0$ and $\left(s, \mu_{t}^{p}(s)\right)>0$ for any $s \in B_{r}(t) \cap \partial \mathscr{K}_{t}^{p}$ with $r$ sufficiently small.

Then let $s \in B_{r}(t) \cap \partial \mathscr{K}_{t}^{p}$; for any $\mu \in B\left(\mu_{t}^{p}(s)\right) \cap \mathscr{H}_{\mu_{t}^{p}(s)}^{s}$, if $\left\{X_{i}\right\}_{i \geq 1}$ is a sequence of independent and identically distributed random vectors distributed according to $Q_{t ; s}^{(p)}$, for $n \in \mathbb{N}$, we can rewrite (38) as

$$
h_{t}^{(p)}(\lfloor n \mu\rfloor)=\delta_{0}(\lfloor n \mu\rfloor)+e^{-\langle\lfloor n \mu\rfloor, s\rangle} \sum_{k \geq 1} \bigotimes_{i=1}^{k} Q_{t ; s}^{(p)}\left\{\sum_{i=1}^{k} X_{i}=\lfloor n \mu\rfloor\right\} .
$$

Then the proof of the Theorem 1 follow from (9).

2.5. The case of the FK random-cluster model. One can extend the previous result to finite connections of the FK random-cluster model when the parameter $p$ related to the occupation probability of a bond is close to 1 . As in the independent percolation case, an estimate on the size of the surface in the dual lattice surrounding a finite cluster of two points, as the one given in Proposition 5, is still in force due to the stochastic domination inequalities given in (19).

This led us to consider only realizations of the cluster $\boldsymbol{C}_{\{0, x\}}$ whose external boundary has size smaller than or equal to $(1+\delta) \varphi(x)$ for sufficiently small $\delta$. Moreover, a suitably modified deterministic statement, as the one presented before to prove mass-gap condition, allows us to give a probabilistic description of $\boldsymbol{C}_{\{0, x\}}$ in terms of a concatenation of subclusters stretching along the direction dual to $\hat{x}$ with respect to $\tau_{p, q}$, whose sizes, under the conditional measure $\mathbb{P}_{q, p}\left(\cdot \mid\left\{0<\left|\boldsymbol{C}_{\{0, x\}}\right|<\infty\right\}\right)$, have exponentially decaying tails. 
When the parameter $p$ is close to 1 , one can perform a polymer expansion for the supercritical random-cluster model. In this way, we can reduce the analysis of the exact asymptotics of the finite two-point connection function to the proof of a local limit theorem result for a random process via thermodynamic formalism as in [Campanino et al. 2003].

We refer the reader to [Campanino and Gianfelice 2015] for the details.

\section{References}

[van den Berg et al. 2006] J. van den Berg, O. Häggström, and J. Kahn, "Some conditional correlation inequalities for percolation and related processes", Random Structures Algorithms 29:4 (2006), $417-435$.

[Campanino and Gianfelice 2009] M. Campanino and M. Gianfelice, "A local limit theorem for triple connections in subcritical Bernoulli percolation”, Probab. Theory Related Fields 143:3-4 (2009), 353-378.

[Campanino and Gianfelice 2011] M. Campanino and M. Gianfelice, "On the Ornstein-Zernike behaviour for the Bernoulli bond percolation on $\mathbb{Z}^{d}, d \geq 3$, in the supercritical regime", J. Stat. Phys. 145:6 (2011), 1407-1422.

[Campanino and Gianfelice 2015] M. Campanino and M. Gianfelice, "On the Ornstein-Zernike behaviour for the supercritical random-cluster model on $\mathbb{Z}^{d}, d \geq 3$ ”, J. Stat. Phys. 159:6 (2015), $1456-1476$.

[Campanino and Ioffe 2002] M. Campanino and D. Ioffe, "Ornstein-Zernike theory for the Bernoulli bond percolation on $\mathbb{Z}^{d}$ ", Ann. Probab. 30:2 (2002), 652-682.

[Campanino et al. 2003] M. Campanino, D. Ioffe, and Y. Velenik, "Ornstein-Zernike theory for finite range Ising models above $T_{c}$ ", Probab. Theory Related Fields 125:3 (2003), 305-349.

[Campanino et al. 2008] M. Campanino, D. Ioffe, and Y. Velenik, "Fluctuation theory of connectivities for subcritical random cluster models", Ann. Probab. 36:4 (2008), 1287-1321.

[Campanino et al. 2010] M. Campanino, D. Ioffe, and O. Louidor, "Finite connections for supercritical Bernoulli bond percolation in 2D", Markov Process. Related Fields 16:2 (2010), 225-266.

[Carlsson and Wainger 1984] H. Carlsson and S. Wainger, "On the multidimensional renewal theorem”, J. Math. Anal. Appl. 100:1 (1984), 316-322.

[Coquille et al. 2014] L. Coquille, H. Duminil-Copin, D. Ioffe, and Y. Velenik, "On the Gibbs states of the noncritical Potts model on $\mathbb{Z}^{2}$ ", Probab. Theory Related Fields 158:1-2 (2014), 477-512.

[Doney 1966] R. A. Doney, “An analogue of the renewal theorem in higher dimensions", Proc. London Math. Soc. (3) 16 (1966), 669-684.

[Edwards and Sokal 1988] R. G. Edwards and A. D. Sokal, "Generalization of the Fortuin-KasteleynSwendsen-Wang representation and Monte Carlo algorithm”, Phys. Rev. D (3) 38:6 (1988), 2009_ 2012.

[Fortuin and Kasteleyn 1972] C. M. Fortuin and P. W. Kasteleyn, "On the random-cluster model, I: Introduction and relation to other models", Physica 57 (1972), 536-564.

[Gallavotti 1972] G. Gallavotti, "The phase separation line in the two-dimensional Ising model", Comm. Math. Phys. 27 (1972), 103-136.

[Greenberg and Ioffe 2005] L. Greenberg and D. Ioffe, "On an invariance principle for phase separation lines”, Ann. Inst. H. Poincaré Probab. Statist. 41:5 (2005), 871-885. 
[Grimmett 1999] G. Grimmett, Percolation, 2nd ed., Grundlehren der mathematischen Wissenschaften 321, Springer, Berlin, 1999.

[Grimmett 2006] G. Grimmett, The random-cluster model, Grundlehren der mathematischen Wissenschaften 333, Springer, Berlin, 2006.

[Ornstein and Zernike 1914] L. S. Ornstein and F. Zernike, "Accidental deviations of density and opalescence at the critical point of a single substance", Nederl. Akad. Wetensch. Proc. 17:2 (1914), 793-806.

[Stam 1969] A. J. Stam, "Renewal theory in $r$ dimensions, I", Compositio Math. 21 (1969), 383399.

Received 1 Apr 2016. Revised 1 May 2016. Accepted 31 May 2016.

MASSIMO CAMPANINO: massimo.campanino@unibo.it

Dipartimento di Matematica, Università degli Studi di Bologna, I-40127 Bologna, Italy

MiCHELE GianfeliCE: gianfelice@mat.unical.it

Dipartimento di Matematica e Informatica, Università della Calabria, Campus di Arcavacata, I-87036 Arcavacata di Rende, Italy 
EDITORIAL BOARD

ANTONIO CARCATERRA

ERIC A. CARLEN

FRANCESCO DELL'ISOLA

RAFFAELE ESPOSITO

ALBERT FANNJIANG

Gilles A. FranCFORT

Pierangelo MARCATI

JEAN-JACQUES MARIGO

PETER A. MARKOWICH

MARTIN OSTOJA-STARZEWSKI

PIERRE SEPPECHER

DAVID J. STEIGMANN

PAUl STEINMANN

PierRe M. SuQueT

MANAGING EDITORS

MICOL AMAR

CORRADO LATTANZIO

ANGELA MADEO

MARTIN OSTOJA-STARZEWSKI

ADVISORY BOARD

ADNAN AKAY

Holm AltenBaCH

MICOL AMAR

HARM ASKES

TEODOR ATANACKOVIĆ

VICTOR BERDICHEVSKY

GUY BOUCHITTÉ

ANDREA BRAIDES

ROBERTO CAMASSA

MAURO CARFORE

ERIC DARVE

FELIX DARVE

ANNA DE MASI

GianPiEtro DEL Piero

EMMANUELE Di BENEDETTO

BERNOLD FIEDLER

IRENE M. GAMBA

DAVID Y. GAO

SERGEY GAVRILYUK

TIMOTHY J. HEALEY

DOMINIQUE JEULIN

ROGER E. KHAYAT

CORRADO LATTANZIO

ROBERT P. LIPTON

ANGELO LUONGO

ANGELA MADEO

JUAN J. MANFREDI

CARLO MARCHIORO

GÉRARD A. MAUGIN

ROBERTO NATALINI PATRIZIO NEFF

ANDREY PIATNITSKI

ERRICO PRESUTTI

MARIO PULVIRENTI

LUCIO RUSSO

Miguel A. F. SANJUAN

PATRICK SElVADURAI

ALEXANDER P. SEYRANIAN

MIROSLAV ŠILHAVÝ

GUIDO SWEERS

ANTOINETTE TORDESILLAS

LEV TRUSKINOVSKY

JUAN J. L. VELÁZQUEZ VINCENZO VESPRI ANGELO VULPIANI msp.org/memocs

Università di Roma "La Sapienza", Italia

Rutgers University, USA

(CO-CHAIR) Università di Roma "La Sapienza", Italia

(TREASURER) Università dell'Aquila, Italia

University of California at Davis, USA

(CO-CHAIR) Université Paris-Nord, France

Università dell'Aquila, Italy

École Polytechnique, France

DAMTP Cambridge, UK, and University of Vienna, Austria

(CHAIR MANAGING EDITOR) Univ. of Illinois at Urbana-Champaign, USA

Université du Sud Toulon-Var, France

University of California at Berkeley, USA

Universität Erlangen-Nürnberg, Germany

LMA CNRS Marseille, France

Università di Roma "La Sapienza", Italia

Università dell'Aquila, Italy

Université de Lyon-INSA (Institut National des Sciences Appliquées), France

(CHAIR MANAGING EDITOR) Univ. of Illinois at Urbana-Champaign, USA

Carnegie Mellon University, USA, and Bilkent University, Turkey

Otto-von-Guericke-Universität Magdeburg, Germany

Università di Roma "La Sapienza", Italia

University of Sheffield, UK

University of Novi Sad, Serbia

Wayne State University, USA

Université du Sud Toulon-Var, France

Università di Roma Tor Vergata, Italia

University of North Carolina at Chapel Hill, USA

Università di Pavia, Italia

Stanford University, USA

Institut Polytechnique de Grenoble, France

Università dell'Aquila, Italia

Università di Ferrara and International Research Center MEMOCS, Italia

Vanderbilt University, USA

Freie Universität Berlin, Germany

University of Texas at Austin, USA

Federation University and Australian National University, Australia

Université Aix-Marseille, France

Cornell University, USA

École des Mines, France

University of Western Ontario, Canada

Università dell' Aquila, Italy

Louisiana State University, USA

Università dell'Aquila, Italia

Université de Lyon-INSA (Institut National des Sciences Appliquées), France University of Pittsburgh, USA

Università di Roma "La Sapienza”, Italia

Université Paris VI, France

Istituto per le Applicazioni del Calcolo "M. Picone", Italy

Universität Duisburg-Essen, Germany

Narvik University College, Norway, Russia

Università di Roma Tor Vergata, Italy

Università di Roma "La Sapienza”, Italia

Università di Roma “Tor Vergata”, Italia

Universidad Rey Juan Carlos, Madrid, Spain

McGill University, Canada

Moscow State Lomonosov University, Russia

Academy of Sciences of the Czech Republic

Universität zu Köln, Germany

University of Melbourne, Australia

École Polytechnique, France

Bonn University, Germany

Università di Firenze, Italia

Università di Roma La Sapienza, Italia

MEMOCS (ISSN 2325-3444 electronic, 2326-7186 printed) is a journal of the International Research Center for the Mathematics and Mechanics of Complex Systems at the Università dell'Aquila, Italy.

Cover image: "Tangle” by $\odot$ John Horigan; produced using the Context Free program (contextfreeart.org).

PUBLISHED BY

7 mathematical sciences publishers

nonprofit scientific publishing

http://msp.org/

(C) 2016 Mathematical Sciences Publishers 
Special issue in honor of

Lucio Russo

Lucio Russo: A multifaceted life

Raffaele Esposito and Francesco dell'Isola

The work of Lucio Russo on percolation Geoffrey R. Grimmett

"Mathematics" and "physics" in the science of harmonics Stefano Isola

From quantum to classical world: emergence of trajectories in a quantum system Rodolfo Figari and Alessandro Teta

Propagation of chaos and effective equations in kinetic theory: a brief survey Mario Pulvirenti and Sergio Simonella

What decides the direction of a current? Christian Maes

A remark on eigenvalue perturbation theory at vanishing isolation distance Fiorella Barone and Sandro Graffi

Some results on the asymptotic behavior of finite connection probabilities in percolation Massimo Campanino and Michele Gianfelice

Correlation inequalities for the Potts model Geoffrey R. Grimmett

Quantum mechanics: some basic techniques for some basic models, I: The models Vincenzo Grecchi

Quantum mechanics: some basic techniques for some basic models, II: The techniques Vincenzo Grecchi

On stochastic distributions and currents

Vincenzo Capasso and Franco Flandoli

A note on Gibbs and Markov random fields with constraints and their moments Alberto Gandolfi and Pietro Lenarda

Quantum mechanics: light and shadows (ontological problems and epistemic solutions) Gianfausto Dell'Antonio

MEMOCS is a journal of the International Research Center for the Mathematics and Mechanics of Complex Systems at the Università dell' Aquila, Italy.

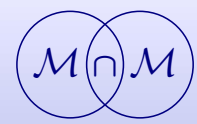

